\title{
The recommendations for pediatric vitreoretinal
}

\section{surgery}

\begin{abstract}
Pediatric vitreoretinal surgery (PVRS) has important differences compared to that in adults. Main differences are on sclerotomy sites, the necessity for posterior hyaloid separation and complete vitrectomy, endo-tamponade, the choice of surgery type and approach according to vitreoretinal disease. Here, we tried to summarize the main differences in PVRS compared to adult vitreoretinal surgery and some critical points for this surgery.
\end{abstract}

Keywords: recommendations, suggests, pediatric, vitreoretinal surgery, children, differences

\author{
Volume 9 Issue 6 - 2019 \\ Burak Turgut,' Tamer Demir,' Onur Çatak, ${ }^{2}$ \\ 'Department of Ophthalmology, Faculty of Medicine, Onsekiz \\ Mart University, Turkey \\ ${ }^{2}$ Department of Ophthalmology, Faculty of Medicine, Firat \\ University, Turkey
}

Correspondence: Burak Turgut, Department of Ophthalmology, Faculty of Medicine, Onsekiz Mart University, Turkey, Tel +90533 7128389, Email burakurgut@comu.edu.tr

Received: September 24, 2019 | Published: November 07, 2019

\section{Introduction}

Pediatric vitreoretinal surgery (PVRS) has important differences compared to that in adults. The main indications for PVRS include rhegmatogenous retinal detachments (RRD) caused by trauma, congenital retinoschisis, Marfan, Stickler or morning-glory syndrome, peripheral retinal degenerations, fundus coloboma, retinoblastoma, myopia, previous ocular surgery and tractional retinal detachments (TRD) caused by retinopathy of prematurity (ROP), persistent fetal vasculature (PFV), familial exudative vitreoretinopathy (FEVR) and penetrating ocular trauma. RRD in the adult usually associates with the posterior vitreous detachment (PVD), while as the RRD in pediatric age is often caused by trauma or congenital abnormalities. As pediatric retinal detachments (RD) usually present in late stages and pediatric ocular anatomy is featured, PVRS is more difficult and complex and has greater complication rates. The most common and devastating complication of PVRS is proliferative vitreoretinopathy (PVR). In particular, creating an iatrogenic retinal break (IRB) during PVRS can cause PVR in children because of the strong inflammatory and proliferative response. ${ }^{1-5}$

\section{Specifical evaluations are required}

Some specifical evaluations such as preoperative planning, appropriate information to parents on the management, good examination and viewing of fundus under general anesthesia, decision on application of scleral buckle (SB) or vitrectomy, the entries, angles and placement of sclerotomy and trocars, crystalline lens status, separation of the posterior hyaloid, selection of intravitreal tamponade and also visual rehabilitation are required for PVRS. ${ }^{6-14}$

\section{The ocular response to surgical trauma in the pediatric age is different}

The ocular response to surgical trauma in the pediatric age differs from those of the adults. Retinal breaks $(\mathrm{RB})$ in children can stimulate strong inflammatory responses and increase the risk of PVR. Thus, core vitrectomies to relieve traction without inducing PVDs may be considered in young children. If possible, retinectomy and posterior drainage retinotomy should be avoided because PVR often develops around retinectomy edges and $\mathrm{RD}$ in pediatric eyes. If a retinotomy is essential, the creation only one retinotomy which is as close to the ora serrata as possible should be performed.$^{6-14}$

\section{To diminish the development of PVR is critical}

To avoid PVR, if possible, it should not enter into vitreous cavity in children, and SB procedure with the formation of circumferential chorioretinal adhesions of RBs using with laser photocoagulation or cryotherapy should be a primary choice the treatment of pediatric primary RRD without markedly tractions or funnel-shaped detachments. The encircling SB relieves vitreoretinal tractions, and the formed vitreous may act as a original endo-tamponade for retinal breaks. ${ }^{6-14}$

\section{A complete posterior hyaloid removal is not always required}

Mechanically and safely creation of a complete posterior hyaloid separation (PHS) or the surgical induction a PVD and completely removing the vitreous is too difficult and these may result in vitreoschisis or IRB in PVRS. In the other hand, the residual vitreous can lead to developing PVR and poor surgical outcomes in pediatric RRD patients. But yet, to try for complete removal of vitreous is not recommended especially in eyes with ROP. ${ }^{6-14}$

Pediatric ocular anatomy has not only some dimensional or proportional but also structural differences compared to the adults. This means "the child's eye is not the small one of the adult eye. In children, the vitreous is densely formed and the cortical vitreous is strongly attached to the internal limiting membrane (ILM). Forceful creation of a PVD often carries a high risk of the IRB. The peripheral vitrectomy can be avoided in cases involving the macula as leaving peripheral vitreous may have a lower risk compared to creating a peripheral break..$^{6-14}$

\section{In pediatric vitreoretinal surgery, water tight closure of sclerotomy} entries is essential

The pediatric sclera has decreased stiffness and thickness, and increased elasticity compared to adults. As children under 2 years old have very thin sclera, the scleral path of trochar is shorter than that 
of the adult. Thus, if the use of the trocar cannula system is planned, it should be considered that they are useful in children only over 2 years old. In pediatric cases, sclerotomies may not be closed properly without sutureless due to scleral elasticity, and this may cause wound leaking, ocular hypotony, retinal, choroidal or vitreous hemorrhage and endophthalmitis. Thus, in pediatric patients, the sutured sclerotomies to provide a water-tight closure of sclerotomy entries but not sutureless sclerotomies is recommended. Closure of the sclera and conjunctiva should be done with absorbable $8-0$ vicryl sutures. ${ }^{6-14}$

\section{The scleral buckle is preferred to PVRS in the management of} most pediatric vitreoretinal diseases

Pediatric vitrectomy has some potential complications such as cataract formation and IRB. In children, vitrectomy usually indices if SB fails. Even if the children have PVR, an SB is also often chosen as the primary treatment option for treatment-naive eyes. As the secondary RRDs to the ROP have usually broad vitreous insertions, the usage of wider SB explants may be recommended. Additionally, an SB procedure may be combined with vitrectomy for traumatic, myopic and peripheral TRDs. In the eyes with pediatric PVR which have not been previously vitrectomized, PVR usually consists of subretinal bands which usually not lead to retinal traction. ${ }^{6-14}$

SB is recommended, especially in trauma-induced posterior segment complications. In complex cases, vitreous base cleaning is essential, so this procedure may need to be done properly for lensectomy. Encircling SB may be helpful to patients with incomplete vitreous removal. SB usually provides good results for the treatment of RBs which are peripheral and reachable with a buckle such as traumatic RD and for RDs associated retinoschisis. An SB may be used as a primary cerclage or as an encircling band in combination with vitrectomy. In the combination with a vitrectomy, a number 40 $(2 \mathrm{~mm})$ or $240(2.5 \mathrm{~mm})$ silicone band as the encircling band in infants may be usually placed just anterior to the equator. In pediatric RRDs, a broad, low buckle with precise localization of the retinal break on the buckle is usually recommended. In RD due to ROP, the band should be placed to support the ridge along the anterior portion of the element. However, as the sclerae in children are thinner than those in adults, scleral belt loops should be avoided. ${ }^{6-14}$

\section{The resorption of subretinal fluid may last for months}

As majority of pediatric RDs are chronic and the resorption of subretinal fluid (SRF) may last for months, and also healthy retinal pigment epithelium (RPE) is a powerful pump for the retinal attachment, draining of SRF in PVRS may not necessary for retinal attachment and persistent SRF is expected at the early period following PVRS. ${ }^{6-14}$

\section{In PVRS, trans-limbal or trans pars plicata/pars plana approach} (trans-vitreal approach) should be chosen according to the case

Although the pars plana approach is more safely, it is usually not fully performed until 8-9 months old, the pars plicata approach is preferred in the newborn eye. The trans pars plicata lens sparing vitrectomy (LSV) can only be considered if the surgeon can safely introduce the instruments without causing an IRB. Thus, this approach may not be possible in patients with Stage 5 ROP with severe anterior TRD and severe PFV with diffuse retrolental plaques. The trans-vitreal approach cannot provide safely dissection of the anterior border of the ciliary body without damaging the crystalline lens in some cases with stage 4 ROP-related RD. In most stage 4A and many stage 4B ROP related RDs, it is possible to relieve traction with LSV. In these cases, the trans-limbal approached vitrectomy with lensectomy is usually preferred, because of the risk of instruments entering the subretinal space. In the RDs in which there is no space in the pars plicata/plana such as in stage 5 ROP, anterior segment dysgenesis or severe PFV, to create entry the eye at limbus or iris root by an anterior chamber infusion is necessary. In the children with advanced $\mathrm{RD}$, lensectomy should include a total capsulectomy because a residual capsule can behave as a scaffold to epiretinal membrane proliferation and it can cause circumferential vitreo-retinal contraction. A total capsulectomy will facilitate the dissection of epiretinal or tractional proliferative membranes away from the retina. If the pediatric vitreoretinal disease cannot affect the crystalline lens, the lens removal can safely be performed. As pars plana may be congenitally abnormal in some cases, trocars should be placed at the limbus for minimizing trocar related damage to pars plana. ${ }^{8,11,13}$

The main goals in PVRS are not to cause IRB and to release traction with peeling membranes and cutting tractional bands

The main purposes of PVRS in RRD include to provide core vitrectomy, relieving of the trans-vitreal or vitreoretinal traction, peeling and removal of the proliferative tissue with minimal intervention and instrumentation. ${ }^{6-14}$ In the treatment of pediatric TRD developed due to ROP, FEVR, and PFV, the main goal of PVRS is the the removal of significant media opacities, peeling membranes and/ or cutting tractional bands and releasing traction. A core vitrectomy but not a complete vitrectomy is carefully performed with attention to the vectors of traction. In pediatric TRD cases, it is recommended the waiting with patience for retinal re-attachment with the spontaneous resorption of SRF over time. As a single IRB developed during infant TRD surgery usually makes the eye inoperable, the main goal of pediatric TRD surgery is the release the traction without causing any IRB. However, in severe TRDs such as stage 5 ROP or FEVR with lens-cornea touch, the anterior chamber reformation, iridectomy, lensectomy and capsulectomy and then the dissection of the plaque and central stalk for providing open the funnel without any IRB should be performed, reversely. The main surgical goal in PFV is to cut the stalk while the that in endophthalmitis is vitrectomy avoiding the aggressive shaving that can cause to IRB in a possibly necrotic or thinned and edematous retina. ${ }^{6,8,11-13}$

\section{Visual and anatomical outcomes vary according to the categories of pediatric RD}

Pediatric RRDs were associated with better visual and anatomical success and conservation of globe, whereas TRDs generally have poorer visual and anatomical outcomes. The pediatric non-traumatic RRD usually present at a later stage while the traumatic RRD usually present acutely compared to adults. The management of RD often lasts for a long because of visual rehabilitation and the fight to amblyopia compared to adults. Compared with adults, pediatric RDs have higher rates of macula-off detachment, proliferative vitreoretinopathy (PVR), chronic duration, and worse visual acuity. The results of vitrectomy for pediatric RDs are generally worse than adults. TRD carries the lowest rate of positive visual outcome while non-traumatic RRDs have the best rates of anatomical success and globe conservation. ${ }^{1-5}$

\section{The risk of crystalline lens injury is higher}

Children have smaller globes, shorter axial lengths and a larger ratio of crystalline lens to the bulbus oculi compared to those of the adult eye. Additionally, pediatric eyes are less developed and they change with age. Thus, the surgeon performing PVRS should be very careful for not hazard the crystalline lens during trocar insertion 
and instrument manipulation. Although the cataract development following PVRS is rare, even only once intraoperatively touch to the crystalline lens may lead to the cataract. The oblique placement of the trocars is associated with an increased risk of accidental lens injury. The best options to avoid the contact to the lens are to use tangential 20 -gauge self-sealing sclerotomies, to create more perpendicularly sclerotomies without trocars or the scleral trocar/cannula entries and insertion of intravitreal instruments to parallel to the visual axis. Additionally, the surgeon should always observe using indirect ophthalmoscopy whether or not there is the aberrant retinal tissue in the projection region the trocars will be placed. ${ }^{6-14}$

\section{Locations of sclerotomy or trocar entries should be chosen to the} age of the child

The pars plana are not fully formed until approximately 8-9 months old. The sclerotomy sites are placed more anteriorly in infants and as a general rule, at $4 \mathrm{~mm}$ from the limbus only in children with 4 years and older. A practical approach, addition $1 \mathrm{~mm}$ to the distance from the limbus for each year of life after the first-year-old can be used. To allow safe sclerotomy, transconjunctival trocar ports should be created with entering $1 \mathrm{~mm}$ posterior to the limbus to perform pars plicata vitrectomy for 1 year, $2 \mathrm{~mm}$ for 2 -year-olds, and $3 \mathrm{~mm}$ posterior to the limbus for 3-year-olds or older children and young adults. Additionally, trans-scleral illumination is a useful method to determine the location of the pars plana for safe sclerotomy entry especially in the eyes without common anatomical features including PFV or severe anterior segment abnormalities. Additionally, it should be reminded to place the trocars at appropriate angle for preventing iatrogenic lens injury because the crystalline lens makes up a large proportion of the eye. ${ }^{6-14}$

In pediatric eyes, the scleral rigidity is lower. Thus, it should be considered that posterior pars plicata LSV may be more appropriate for pediatric cases. The sclerotomy sites should be placed more anteriorly in infants, the crystalline lenses should be removed, and shorter instruments should be used. Insertion sites at the limbus or cornea with the removal of the crystalline lenses may be beneficial in premature eyes with anterior pathologies; however, aphakic patients are at risk for developing amblyopia and glaucoma. An alternative is to perform LSV; entering at more perpendicular angles and using shorter cannulas may decrease potential damage to the lens and surrounding retina. Inserting cannulas through stacked silicone bands can decrease the intraocular lengths. ${ }^{6-14}$

Silicone oil tamponade may be used in the most pediatric RD cases excluding stage 5 ROP, advanced posterior PFV, and fundus coloboma

As the children have usually complicated RDs and often can not comply with appropriate head positioning for tamponing following PVRS, 5,000 centistokes silicone oil as intravitreal tamponade is preferred to intraocular gase. However, in children with advanced RD such as in stage 5 ROP or advanced posterior PFV with RD and coloboma with RD (the increased risk of subretinal migration), the silicone oil as intravitreal tamponade should be avoided. ${ }^{6-14}$

\section{Perfluorocarbon (PFCL)s are not usually preferred in children}

In adults, the creation of retinotomies or retinectomies and the usage of perfluorocarbon liquids for flattening the retina can usually be performed while as these are not preferred in children because to provide completely flatten the retina. If the usage of PFCL in PVRS is unavoidable, perfluorodecalin having the highest gravity may be preferred for flattening usually thicker retinae. ${ }^{6-14}$

\section{Enzymatic or pharmacologic vitreolysis may facilitate PVRS in complicated pediatric vitreoretinopathies}

Pharmacologic or enzymatic vitreolysis (PVL) using with intravitreal ocriplasmin, microplasmin, autologous plasmin enzymes may be performed preoperatively to facilitate the PHS, to remove vitreous and to reduce the risk of intraoperative IRBs in complicated pediatric vitreoretinopathies in which a PHS is highly necessary such as stage 5 ROP, congenital X-linked retinoschisis (CXLRS) and pediatric traumatic macular hole. It is considered that PVL weaks the vitreo-retinal junction, so, facilitates PHS and diminishes the risk of the retina trauma and IRBs. Additionally, PVL may facilitate the removal of epiretinal proliferative membranes reducing the risk of IRB during epiretinal membrane peeling in above mentioned complicated or advanced cases. The intravitreal injection of agents for PVL is often performed after induction of general anesthesia, and approximately 30 minutes before the start of surgery in the operating room but not in the clinic. ${ }^{6-14}$

\section{Other considerations}

A. Currently, 23G or $25 \mathrm{G}$ vitrectomy instruments are mostly used in PVRS. However, the short in length 25 or $27 \mathrm{G}$ instruments may be more adequate for surgery in PVRS because globes are very small and these systems can cause potentially fewer IRBs. ${ }^{1-14}$

B. Use of the labeled or off-label triamcinolone acetonide injectable suspension can provide better visualization of the posterior hyaloid. ${ }^{1-14}$

C. In eyes with PVR, to create the segmentation of membrane should be preferred to delamination for removing pre-retinal membranes because of tight vitreoretinal attachments. ${ }^{6-14}$

D. Same-day bilateral PVRS should be considered only in selected patients such as the cases who are not adequate to undergone two separate surgery because of transporting difficulty, the emergency of intervention, or risk of amblyopia or who can not tolerate two general anesthesia session because of medical reasons such as higher mortality. ${ }^{6-14}$

E. Compared to adults, children have lower systolic blood pressure. Thus, it should be kept on mind that the high elevation in infusion pressure or prolonged scleral depression can lead to developing iatrogenic occlusion of the central retinal artery. Therefore, the optic nerve and vessel pulsations should be monitored during continually in PVRS. ${ }^{6-14}$

F. Postoperative monitoring and treatment of amblyopia are essential in maximizing functional outcomes in pediatric patients. After surgery, refractive error in the child should be corrected and amblyopia should be treated aggressively. Additionally, it should also be reminded that long-acting gas tamponade or silicone oil can cause deprivation amblyopia in the child. ${ }^{6-14}$

G. Mono-ocular children and parents must be warned regarding avoiding contact sports, and the usage of polycarbonate protective lenses and special spectacles for sports to avoid accidental ocular trauma to the healthy eye..$^{6-14}$ 


\section{Conclusion}

There are some differences in PVRS compared to adult vitreoretinal surgery and some critical points for this surgery. To know and remind these may diminish undesirable and dramatical consequences of PVRS.

\section{Disclosures}

The authors declare that there is no conflict of interest regarding the publication of this paper.

\section{Compliance with ethics}

This study involves a review of the literature and did not involve any studies with human or animal subjects performed by any of the authors.

\section{Authorship}

All authors have contributed to the concept and design, data collection, literature Search in the work and writing of the manuscript. The named authors meet the International Committee of Medical Journal Editors (ICMJE) criteria for authorship of this manuscript, take responsibility for the integrity of the work as a whole, and have given final approval to the version to be published.

\section{References}

1. Al-Zaaidi S, Al-Rashaed S, Al-Harthi E, et al. Rhegmatogenous retinal detachment in children 16 years of age or younger. Clin Ophthalmol. 2013;7:1001-1014.

2. Wadhwa N, Venkatesh P, Sampangi R, et al. Rhegmatogenous retinal detachments in children in India: clinical characteristics, risk factors, and surgical outcomes. J AAPOS. 2008;12(6):551-554.
3. Wenick AS, Barañano DE. Evaluation and management of pediatric rhegmatogenous retinal detachment. Saudi J Ophthalmol. 2012;26(3):255-263.

4. Read SP, Aziz HA, Kuriyan A, et al. Retinal detachment surgery in a pediatric population: visual and anatomic outcomes. Retina. 2018;38(7):1393-1402.

5. Gan NY, Lam WC. Retinal detachments in the pediatric population. Taiwan J Ophthalmol. 2018;8(4):222-236.

6. Yonekawa Y, Fine HF. Practical Pearls in Pediatric Vitreoretinal Surgery. Ophthalmic Surg Lasers Imaging Retina. 2018;49(8):561-565.

7. Leung EH, Berrocal AM. Pediatric Microincision Vitreoretinal Surgery. Int Ophthalmol Clin. 2016;56(4):203-208.

8. Gan NY, Lam WC. Special considerations for pediatric vitreoretinal surgery. Taiwan J Ophthalmol. 2018;8(4):237-242.

9. Hu Y, Tang S. Major challenges in vitreoretinal surgery. Taiwan J Ophthalmol. 2014;5(1):9-14.

10. Sebag J. Age-related differences in the human vitreoretinal interface. Arch Ophthalmol. 1991;109(7):966-971.

11. Ranchod TM, Capone A Jr. Tips and tricks in pediatric vitreoretinal surgery. Int Ophthalmol Clin. 2011;51(1):173-183.

12. Baumal CR, Berrocal AM. Pearls for Pediatric Retinal Surgery. Retina Today. 2018. SE

13. Nudleman E, Capone Jr A. Management of Complicated Vitreoretinal Cases in Children. In: Patelli F, Stanislao R, editors. Management of Complicated Vitreoretinal Diseases. Switzerland: Springer International Publishing; 2015. p. 21-30.

14. Wright LM, Harper CA, Chang EY. Management of Infantile and ChildhoodE Retinopathies: Optimized Pediatric Pars Plana Vitrectomy Sclerotomy Nomogram. Ophthalmology Retina. 2018;2(12):1227-1234. 